\title{
Stability analysis for boundary value problems with generalized nonlocal condition via Hilfer-Katugampola fractional derivative
}

\author{
Idris Ahmed ${ }^{1,2,3}$, Poom Kumam ${ }^{2,4^{*}}$ (D) Fahd Jarad ${ }^{5}$, Piyachat Borisut ${ }^{1,2}$, Kanokwan Sitthithakerngkiet $^{6}$ and \\ Alhassan Ibrahim ${ }^{7}$
}

\author{
"Correspondence: \\ poom.kum@kmutt.ac.th \\ ${ }^{2}$ Center of Excellence in Theoretical \\ and Computational Science \\ (TaCS-CoE), Science Laboratory \\ Building, King Mongkut's University \\ of Technology Thonburi (KMUTT), \\ Bangkok, Thailand \\ ${ }^{4}$ Department of Medical Research, \\ China Medical University Hospital, \\ China Medical University, Taichung, \\ Taiwan \\ Full list of author information is \\ available at the end of the article
}

\begin{abstract}
In this research, we present the stability analysis of a fractional differential equation of a generalized Liouville-Caputo-type (Katugampola) via the Hilfer fractional derivative with a nonlocal integral boundary condition. Besides, we derive the relation between the proposed problem and the Volterra integral equation. Using the concepts of Banach and Krasnoselskii's fixed point theorems, we investigate the existence and uniqueness of solutions to the proposed problem. Finally, we present two examples to clarify the abstract result.
\end{abstract}

MSC: $26 \mathrm{~A} 33 ; 34 \mathrm{~A} 34 ; 34 \mathrm{~B} 15$

Keywords: Hilfer fractional derivative; Stability; Volterra integral equation; Nonlocal integral condition

\section{Introduction}

Fractional calculus is a branch of mathematics that examines the properties of derivatives and integrals of arbitrary non-integer order. This theory has been studied over the years and was initiated in a letter written to Guillaume de l'Hopital by Gottfried Wilhelm Leibniz in 1965. Subsequently, the area has become a great area of interest to many famous mathematicians such as Fourier, Laplace, Abel, Liouville, Riemann, Letnikov etc. For more interpretation on the fundamentals ideas of fractional calculus, physical and geometric meanings we refer the reader to see the books, $[29,37,39,54]$ etc.

There are various definitions of fractional order derivatives and integrals, for example, we have the Riemann, Liouville, Caputo, Riemann-Liouville, Weyl, Hadamard, and Grunwald-Letnikov versions. These derivatives do not coincide in general except for some special cases. This is due to the fact that the authors try to preserve some properties of these operators. However, the most famous definitions are the ones for Riemann, Liouville, Riemann-Liouville, and Caputo fractional order derivatives and integrals.

(c) The Author(s) 2020. This article is licensed under a Creative Commons Attribution 4.0 International License, which permits use, sharing, adaptation, distribution and reproduction in any medium or format, as long as you give appropriate credit to the original author(s) and the source, provide a link to the Creative Commons licence, and indicate if changes were made. The images or other third party material in this article are included in the article's Creative Commons licence, unless indicated otherwise in a credit line to the material. If material is not included in the article's Creative Commons licence and your intended use is not permitted by statutory regulation or exceeds the permitted use, you will need to obtain permission directly from the copyright holder. To view a copy of this licence, visit http://creativecommons.org/licenses/by/4.0/. 
Fractional differential equations are an area that is related to the fractional calculus and it played an important role in numerous fields of engineering and scientific areas such as physics, chemistry, biology, economics, control theory, signal and image processing. Recently, it was proved that fractional derivatives and integrals of arbitrary order are more advantageous and pragmatic for characterizing the memory and hereditary properties of different materials and processes than the integer-order derivatives; see [14, 18, 32].

Existence, uniqueness and stability analyses of fractional differential equations with different types of initial and boundary conditions belong to the most essential parts in the theory of fractional differential equations to which this paper is devoted. However, many researchers studied the existence and uniqueness of solutions for boundary value problems (BVPs) of fractional differential equations with different types of fractional integrals and derivatives; see for example [5, 7, 13, 17, 25, 33, 36, 40-43, 45, 52, 55]. Hilfer initiated a new class of Riemann-Liouville fractional derivatives, called Hilfer fractional derivatives. These fractional derivatives coincide with the classical Riemann-Liouville and Caputo fractional derivatives. The main advantage of these operators is the degree of freedom on the initial conditions. Thus, based on this setting, it motivated a lot of researchers to initiate research and finding the existence, uniqueness and stability results; see [2$4,8,10,15,16,21,27,48,50]$.

More recently, motivated by Hilfer fractional derivatives and some well-known results in respect of the generalized classical definitions of Riemann-Liouville and Caputo fractional derivatives, Oliveira and Capelas [35] proposed new fractional derivatives called HilferKatugampola fractional derivatives, which incorporate Hilfer and generalized fractional derivatives. These have been contemplated by some researchers in the field of fractional calculus, results can be found in $[1,9,20,23,24,26,28,53]$ and the references therein. Stability analysis is one of the most important aspects of fractional differential equations. This notion was initiated by Ulam [22], which motivated many researchers to make contributions in this area $[6,8,10,11,21,28,31,38,46,47,51]$. For instance, Ahmed et al. [9] developed the existence and uniqueness criteria for solutions of boundary value problems (BVPs) with a generalized nonlocal integral and multi-point conditions described by

$$
\left\{\begin{array}{l}
{ }^{\rho} \mathcal{D}_{0^{+}}^{v} z(s)=g(s, z(s)), \quad s \in \mathcal{J}=(0, T], s>0 \\
\delta z(0)=\sum_{j=1}^{k} d_{j} z\left(\lambda_{j}\right), \quad z(T)=\sum_{i=1}^{m} b_{i}{ }^{\rho} \mathcal{I}_{0^{+}}^{q} z\left(\xi_{i}\right)+c, \quad \xi_{i}, \lambda_{j} \in \mathcal{J}, c, b_{i}, d_{j} \in \mathbb{R}, \\
0<\xi_{1}<\cdots<\xi_{i}<\cdots<\xi_{m}<\lambda_{1}<\cdots<\lambda_{j}<\cdots<\lambda_{k}<T
\end{array}\right.
$$

and the inclusion problem

$$
\left\{\begin{array}{l}
{ }^{\rho} \mathcal{D}_{0^{+}}^{v} z(s) \in G(s, z(s)), \quad s \in \mathcal{J}=(0, T], s>0, \\
\delta z(0)=\sum_{j=1}^{k} d_{j} z\left(\lambda_{j}\right), \quad z(T)=\sum_{i=1}^{m} b_{i}{ }^{\rho} \mathcal{I}_{0^{+}}^{q} z\left(\xi_{i}\right)+c, \quad \xi_{i}, \lambda_{j} \in \mathcal{J}, \\
0<\xi_{1}<\cdots<\xi_{i}<\cdots<\xi_{m}<\lambda_{1}<\cdots<\lambda_{j}<\cdots<\lambda_{k}<T,
\end{array}\right.
$$

where ${ }^{\rho} \mathcal{D}_{0^{+}}^{\nu}(\cdot)$ is the generalized fractional derivatives of order $(1<v \leq 2)$ and ${ }^{\rho} \mathcal{I}_{0^{+}}^{q}(\cdot)$ is a generalized fractional integral of order $q>0, \delta=s^{1-\rho} \frac{d}{d s}, \rho>0, g:(0, T] \times \mathbb{R} \rightarrow \mathbb{R}$ is a continuous function, $T>0$ and $G: \mathcal{J} \times \mathbb{R} \rightarrow \mathbb{P}(\mathbb{R})$ is a multivalued function. Recently, Suphawat et al. [12] initiated research and found existence and uniqueness results for the boundary value problems (BVPs) which involve Hilfer fractional derivatives and a nonlocal 
condition described by

$$
\left\{\begin{array}{l}
{ }^{H} \mathcal{D}_{a^{+}}^{p, q} w(s)=\Theta(s, w(s)), \quad s \in \mathcal{I}=[a, b], b>a>0 \\
w(a)=0, \quad w(b)=\sum_{k=1}^{m} b_{k} \mathcal{I}_{a^{+}}^{r_{k}}\left(\xi_{k}\right), \quad \xi_{k} \in \mathcal{I}, b_{k} \in \mathbb{R} \\
a<\xi_{1}<\cdots<\xi_{i}<\cdots<\xi_{k}<b
\end{array}\right.
$$

where ${ }^{H} \mathcal{D}_{a^{+}}^{p, q}(\cdot)$ is the Hilfer fractional derivatives of order $(1<p<2)$ and the parameter $0 \leq q \leq 1, \mathcal{I}_{a^{+}}^{r_{k}}(\cdot)$ is the Riemann-Liouville fractional integral of order $r_{k}>0, \Theta: \mathcal{I} \times \mathbb{R} \rightarrow$ $\mathbb{R}$ is a continuous function. The authors employed a fixed point approach of the Banach contraction principle, and the Hölder, Boyd and Wang, Leray-Schauder and Krasnoselskii's approaches to obtain their results.

In [20], Harikrishnan considered an initial value problem with the nonlocal condition given by

$$
\left\{\begin{array}{l}
{ }^{\rho} D_{a^{+}}^{v, q} u(\tau)=f(\tau, u(\tau)), \quad \tau \in I=(a, T] \\
{ }^{\rho} I_{a^{+}}^{1-r} u(a)=\sum_{j=1}^{n} c_{j} u\left(\sigma_{j}\right), \quad \sigma_{j} \in I
\end{array}\right.
$$

where ${ }^{\rho} D_{a^{+}}^{v, q}(\cdot)$ is the Hilfer-Katugampola fractional derivative of order $(0<v<1)$ and parameter $0 \leq q \leq 1,{ }^{\rho} I_{a^{+}}^{1-r}(\cdot)$ is the generalized fractional integral of order $1-r>0, \rho>0$, $f: I \times \mathbb{R} \rightarrow \mathbb{R}$ is a continuous function, $c_{j} \in \mathbb{R}$ and $\sigma_{j} \in I$ satisfying $0<a<\sigma_{1} \leq \sigma_{2} \leq \cdots \leq$ $\sigma_{n}<T$ for $j=1,2, \ldots, n$. Existence results were obtained using Krasnoselskii's fixed point theorem.

More recently, Vivek et al.[48] studied an implicit differential equation with nonlocal condition via Hilfer fractional derivatives described by

$$
\left\{\begin{array}{l}
D_{0^{+}}^{\alpha, \beta} v(s)=\Theta\left(s, v(s), D_{0^{+}}^{\alpha, \beta} v(s)\right), \quad s \in \mathcal{T}=[0, b], b>0, \\
I_{0^{+}}^{1-r} v(0)=\sum_{i=1}^{m} e_{i} v\left(\tau_{i}\right), \quad \tau_{i} \in \mathcal{T}, r=\alpha+\beta-\alpha \beta
\end{array}\right.
$$

where $D_{0^{+}}^{\alpha, \beta}(\cdot)$ is the Hilfer fractional derivatives of order $(0<\alpha<1)$ and type $0 \leq \beta \leq 1$, $I_{0^{+}}^{1-r}(\cdot)$ is the Riemann-Liouville fractional integral of order $1-r>0, \Theta: \mathcal{T} \times \mathbb{R}^{2} \rightarrow \mathbb{R}$ is a continuous function, $e_{i} \in \mathbb{R}, \tau_{i} \in \mathcal{T}$ satisfying $0<\tau_{1} \leq \cdots \leq \tau_{m}<b$. The authors proved the existence and uniqueness via Schafer's and Banach's fixed point theorems. Furthermore, stability results were also investigated.

Motivated by the above series of papers and some basic ideas of the fractional calculus, the main objectives of this paper is to extend the results of $[9,12,19]$ and also to discuss the stability analysis in the framework of Ulam-Hyers and generalized Ulam-Hyers theory. More specifically, we consider the fractional differential equation given by

$$
\left\{\begin{array}{l}
\rho \mathfrak{D}_{a^{+}}^{p, q} x(t)=f(t, x(t)), \quad t \in[a, b], b>a>0, \rho>0, \\
x(a)=0, \quad x(b)=\sum_{i=1}^{m} \mu_{i}^{\rho} \Im_{a^{+}}^{\xi_{i}} x\left(\delta_{i}\right)+\varrho, \quad \xi_{i}>0, \varrho, \mu_{i} \in \mathbb{R}, \delta_{i} \in(a, b),
\end{array}\right.
$$

where ${ }^{\rho} \mathfrak{D}_{a^{+}}^{p, q}(\cdot)$ is the Hilfer-Katugampola fractional derivative of order $(1<p \leq 2)$ and parameter $0 \leq q \leq 1,{ }^{\rho} \Im_{a^{+}}^{\xi_{i}}(\cdot)$ is the generalized fractional integral of order $\xi_{i}>0, f:[a, b] \times$ $\mathbb{R} \rightarrow \mathbb{R}$ is a continuous function, $\mu_{i} \in \mathbb{R}$ and $\delta_{i} \in[a, b]$ satisfying $0<a<\delta_{1} \leq \delta_{2} \leq \cdots \leq$ $\delta_{m}<b$ for $i=1,2, \ldots, m$. Problem (1.6) is simply called a boundary value problem (BVP). 
Evidently, to the best of our knowledge, stability analysis of boundary value problems (BVPs) is still in the initial stage. Thus the main contribution of this paper is to investigate the stability analysis of Ulam-Hyers and generalized Ulam-Hyers theory, which is not established in $[9,12,19]$. Besides, we also show the equivalence between problem (1.6) and the Volterra integral equation and apply the Banach and Krasnoselskii's fixed point theorems to establish the existence and uniqueness results. Moreover, problem (1.6) coincides with:

- the Riemann-Liouville and Hadamard fractional derivative when $q=0, \rho \rightarrow 1$;

- the Hilfer and Hilfer-Hadamard fractional derivative $\left(\rho \rightarrow 1\right.$, and $\left.\rho \rightarrow 0^{+}\right)$;

- the generalized (defined by Katugampola) and Caputo-type versions $(q=0)$ and $(q=1)$.

We organize the remaining part of the paper as follows: Some preliminary facts, vital definitions, and theoretical results are recalled in Sect. 2. In Sect. 3, we derive the relation between the Volterra integral equation and the proposed problem (1.6). Also, with the help of these equations, the existence and uniqueness of solutions are proven using the concepts of the Banach and Krasnoselskii fixed point theorems. In Sect. 4, we present certain criteria under which the proposed problem (1.6) is both Ulam-Hyers and generalized Ulam-Hyers stable. Furthermore, as an application, two examples are given to show the applicability of the obtained results. Finally, we summarize the paper in the last section.

\section{Preliminaries and theoretical results}

This section summarizes the basic definitions and principles of fractional derivatives and integrals and describes the theoretical findings that are useful in this paper. For further information, see $[29,35,37]$.

Definition 2.1 Let $-\infty<a<t<b<\infty, n-1<p<n$ with $n \in \mathbb{N}, n=[p]+1$ and $\rho>0$. Then

$$
\left({ }^{\rho} \mathfrak{I}_{a^{p}}^{p} f\right)(t)=\frac{1}{\Gamma(p)} \int_{a}^{t}\left(\frac{t^{\rho}-\tau^{\rho}}{\rho}\right)^{p-1} \tau^{\rho-1} f(\tau) d \tau
$$

which is referred to as a left-sided generalized fractional integral of order $p$ provided the right side is point-wise defined on $(a, \infty) ; \Gamma(\cdot)$ denotes the gamma function.

Likewise, the generalized fractional derivative is defined in terms of the generalized fractional integral (2.1),

$$
\begin{aligned}
\left({ }^{\rho} \mathfrak{D}_{a^{+}}^{p} f\right)(t) & =\left(t^{1-\rho} \frac{d}{d t}\right)^{n}\left({ }^{\rho} \mathfrak{I}_{a^{+}}^{n-p} f\right)(t) \\
& =\frac{1}{\Gamma(n-p)}\left(t^{1-\rho} \frac{d}{d t}\right)^{n} \int_{a}^{t}\left(\frac{t^{\rho}-\tau^{\rho}}{\rho}\right)^{n-p+1} \tau^{\rho-1} f(\tau) d \tau
\end{aligned}
$$

Definition 2.2 Suppose $n-1<p \leq n, 0 \leq q \leq 1$ with $n \in \mathbb{N}$ and $\rho>1$. Then the fractional operator

$$
\left({ }^{\rho} \mathfrak{D}_{a^{+}}^{p, q} f\right)(t)=\left({ }^{\rho} \mathfrak{I}_{a^{+}}^{q(n-p)}\left(t^{1-\rho} \frac{d}{d t}\right)^{n} \rho \mathfrak{I}_{a^{+}}^{(1-q)(n-p)} f\right)(t)
$$


is called the left-sided Hilfer generalized (or simply Hilfer-Katugampola) fractional derivative of order $p$ and parameter $q$.

Remark 2.3 The operator ${ }^{\rho} \mathfrak{D}_{a^{+}}^{p, q}$ can be further simplified as

$$
\begin{aligned}
\left({ }^{\rho} \mathfrak{D}_{a^{+}}^{p, q} f\right)(t) & =\left({ }^{\rho} \mathfrak{I}_{a^{+}}^{q(n-p)}\left(t^{1-\rho} \frac{d}{d t}\right)^{n} \rho \mathfrak{I}_{a^{+}}^{(1-q)(n-p)} f\right)(t) \\
& ={ }^{\rho} \mathfrak{I}_{a^{+}}^{q(2-p)} \rho \mathfrak{D}_{a^{+}}^{r},
\end{aligned}
$$

where $r=p+q(2-p), 1<p \leq 2,0 \leq q \leq 1$ and the operators ${ }^{\rho} \mathfrak{I}_{a^{+}},{ }^{\rho} \mathfrak{D}_{a^{+}}$are defined in (2.1) and (2.2), respectively.

Next, we consider some important properties of these operators as follows.

Lemma 2.4 Let $p, \theta>0,1 \leq p<\infty, 0<a<b<\infty$ and $\rho, c \in \mathbb{R}$ such that $\rho \geq c$. Then, for $f \in X_{c}^{p}(a, b)$, the following relations hold:

$$
\left({ }^{\rho} \mathfrak{I}_{a^{+}}^{p}{ }^{\rho} \mathfrak{I}_{a^{+}}^{\theta} f\right)(\cdot)=\left({ }^{\rho} \mathfrak{I}_{a^{+}}^{p+\theta} f\right)(\cdot)
$$

and

$$
\left({ }^{\rho} \mathfrak{D}_{a^{+}}^{p}{ }^{\rho} \mathfrak{I}_{a^{+}}^{p} f\right)(\cdot)=f(\cdot)
$$

Lemma 2.5 In view of Eqs. (2.1) and (2.2), for any $p, \theta \geq 0$, we have

$$
\begin{aligned}
& \left(\rho \widetilde{\mathfrak{I}}_{a^{+}}^{p}\left(\frac{t^{\rho}-a^{\rho}}{\rho}\right)^{\theta-1}\right)(x)=\frac{\Gamma(\theta)}{\Gamma(p+\theta)}\left(\frac{t^{\rho}-a^{\rho}}{\rho}\right)^{p+\theta-1}, \\
& \left({ }^{\rho} \mathfrak{D}_{a^{+}}^{p}\left(\frac{t^{\rho}-a^{\rho}}{\rho}\right)^{p-1}\right)(x)=0, \quad 0<p<1 .
\end{aligned}
$$

Lemma 2.6 If $1<p \leq 2$, then

$$
{ }^{\rho} \mathfrak{I}_{a^{+}}^{p}\left({ }^{\rho} \mathfrak{D}_{a^{+}}^{p} f\right)(t)=f(t)-\frac{\left(\rho \mathfrak{I}^{1-r} f\right)(a)}{\Gamma(r)}\left(\frac{t^{\rho}-a^{\rho}}{\rho}\right)^{r-1}-\frac{\left(\rho \mathfrak{I}^{2-r} f\right)(a)}{\Gamma(r-1)}\left(\frac{t^{\rho}-a^{\rho}}{\rho}\right)^{r-2} .
$$

\section{Main results}

In this section, firstly, we establish the relationship between the proposed problem (1.6) and the Volterra integral equation. Secondly, with the help of these equation, we derive the existence and uniqueness of solutions based on Banach's and Krasnoselskii's fixed point theorems.

\subsection{Equivalence with the Volterra integral equation}

We consider the generalized boundary value problem described by

$$
{ }^{\rho} \mathfrak{D}_{a^{+}}^{p, q} x(t)=f(t, x(t)), \quad b \in[a, b], b>a>0,
$$


where $p \in(1,2], q \in[0,1], \rho>0$, subject to the boundary conditions:

$$
x(a)=0, \quad x(b)=\sum_{i=1}^{m} \mu_{i}{ }^{\rho} \Im_{a^{+}}^{\xi_{i}} x\left(\delta_{i}\right)+\varrho, \quad \xi_{i}>0, \varrho, \mu_{i} \in \mathbb{R}, \delta_{i} \in(a, b) .
$$

The following lemma shows the relationship between problem (3.1)-(3.2) and the Volterra integral equation of the form

$$
\begin{aligned}
x(t)= & \frac{\rho^{1-r}}{\Upsilon \Gamma(r)}\left(t^{\rho}-a^{\rho}\right)^{r-1}\left(\rho \mathfrak{I}_{a^{+}}^{p} f(s, x(s))(b)-\sum_{i=1}^{m} \mu_{i}^{\rho} \mathfrak{I}_{a^{+}}^{p+\xi} f(s, x(s))\left(\delta_{i}\right)-\varrho\right) \\
& +{ }^{\rho} \mathfrak{I}_{a^{+}}^{p} f(s, x(s))(t),
\end{aligned}
$$

where

$$
\Upsilon=\sum_{i=1}^{m} \frac{\mu_{i} \rho^{1-\left(r+\xi_{i}\right)}}{\Gamma\left(r+\xi_{i}\right)}\left(\delta_{i}^{\rho}-a^{\rho}\right)^{r+\xi_{i}-1}-\frac{\rho^{1-r}}{\Gamma(r)}\left(b^{\rho}-a^{\rho}\right)^{r-1} \neq 0 .
$$

Lemma 3.1 Let $1<p \leq 2,0 \leq q \leq 1$ such that $r=p+2 q-p q$ and $f(\cdot, x(\cdot)) \in \mathcal{C}([a, b] \times \mathbb{R})$. Then $x$ is a solution of the problems (3.1), (3.2) if and only if it satisfies Eq. (3.3).

Proof Indeed, in view of (2.4), Eq. (3.1) can be written as

$$
\rho \mathfrak{I}_{a^{+}}^{q(2-p) \rho} \mathfrak{D}^{r} a^{+} x(t)=f(t, x(t)) .
$$

Applying the operator ${ }^{\rho} \mathfrak{I}_{a^{+}}^{p}$ to both sides of Eq. (3.5) and using Lemma 2.6, we get

$$
x(t)=\frac{\left(\rho \mathfrak{I}_{a^{+}}^{1-r} x\right)(a)}{\Gamma(r)}\left(\frac{t^{\rho}-a^{\rho}}{\rho}\right)^{r-1}+\frac{\left(\rho \mathfrak{I}_{a^{+}}^{2-r} x\right)(a)}{\Gamma(r-1)}\left(\frac{t^{\rho}-a^{\rho}}{\rho}\right)^{r-2}+{ }^{\rho} \mathfrak{I}_{a^{+}}^{p} f(t, x(t)) .
$$

Denoting $e_{1}=\left({ }^{\rho} \mathfrak{I}_{a^{+}}^{2-r} X\right)(a)$ and $e_{2}=\left(\rho \mathfrak{I}_{a^{+}}^{1-r} x\right)(a)$ in Eq. (3.6) yields

$$
x(t)=\frac{e_{2}}{\Gamma(r)}\left(\frac{t^{\rho}-a^{\rho}}{\rho}\right)^{r-1}+\frac{e_{1}}{\Gamma(r-1)}\left(\frac{t^{\rho}-a^{\rho}}{\rho}\right)^{r-2}+{ }^{\rho} \mathfrak{I}_{a^{+}}^{p} f(t, x(t)) .
$$

By substituting the boundary condition $x(a)=0$ in Eq. (3.7), we get $e_{1}=0$. Thus

$$
x(t)=\frac{e_{2}}{\Gamma(r)}\left(\frac{t^{\rho}-a^{\rho}}{\rho}\right)^{r-1}+{ }^{\rho} \mathfrak{I}_{a^{+}}^{p} f(t, x(t)) .
$$

Substituting $t=\delta_{i}$ and multiplying through by $\mu_{i}$ in Eq. (3.8), we have

$$
\mu_{i} x\left(\delta_{i}\right)=\frac{\mu_{i} e_{2}}{\Gamma(r)}\left(\frac{\delta_{i}^{\rho}-a^{\rho}}{\rho}\right)^{r-1}+\mu_{i}^{\rho} \Im_{a^{+}}^{p} f\left(\delta_{i}, x\left(\delta_{i}\right)\right) .
$$

Applying ${ }^{\rho} \mathfrak{I}_{a^{+}}^{\xi_{i}}$ to both sides of Eq. (3.9) and using Lemmas 2.5 and 2.4 yield

$$
\mu_{i}^{\rho} \Im_{a^{+}}^{\xi_{i}} x\left(\delta_{i}\right)=\frac{\mu_{i} e_{2}}{\Gamma\left(r+\xi_{i}\right)}\left(\frac{\delta_{i}^{\rho}-a^{\rho}}{\rho}\right)^{r+\xi_{i}-1}+\mu_{i}{ }^{\rho} \widetilde{I}_{a^{+}}^{p+\xi_{i}} f\left(\delta_{i}, x\left(\delta_{i}\right)\right),
$$


which implies that

$$
\begin{aligned}
\sum_{i=1}^{m} \mu_{i}{ }^{\rho} \mathfrak{I}_{a^{+}}^{\xi_{i}} x\left(\delta_{i}\right)+\varrho= & e_{2} \sum_{i=1}^{m} \frac{\mu_{i}}{\Gamma\left(r+\xi_{i}\right)}\left(\frac{\delta_{i}^{\rho}-a^{\rho}}{\rho}\right)^{r+\xi_{i}-1} \\
& +\sum_{i=1}^{m} \mu_{i}{ }^{\rho} \Im_{a^{+}}^{p+\xi_{i}} f\left(\delta_{i}, x\left(\delta_{i}\right)\right)+\varrho .
\end{aligned}
$$

Also,

$$
x(b)=\frac{e_{2}}{\Gamma(r)}\left(\frac{b^{\rho}-a^{\rho}}{\rho}\right)^{r-1}+{ }^{\rho} \mathfrak{I}_{a^{+}}^{p} f(b, x(b)) .
$$

From the boundary condition $x(b)=\sum_{i=1}^{m} \mu_{i}^{\rho} \mathfrak{\Im}_{a^{+}}^{\xi_{i}} x\left(\delta_{i}\right)+\varrho$ and in view of Eqs. (3.11), (3.12), we obtain

$$
\begin{aligned}
\frac{e_{2}}{\Gamma(r)}\left(\frac{b^{\rho}-a^{\rho}}{\rho}\right)^{r-1}+{ }^{\rho} \mathfrak{I}_{a^{+}}^{p} f(b, x(b))= & e_{2} \sum_{i=1}^{m} \frac{\mu_{i}}{\Gamma\left(r+\xi_{i}\right)}\left(\frac{\delta_{i}^{\rho}-a^{\rho}}{\rho}\right)^{r+\xi_{i}-1} \\
& +\sum_{i=1}^{m} \mu_{i}{ }^{\rho} \Im_{a^{+}}^{p+\xi_{i}} f\left(\delta_{i}, x\left(\delta_{i}\right)\right)+\varrho
\end{aligned}
$$

which implies that

$$
e_{2}=\frac{1}{\Upsilon}\left(\rho \mathfrak{J}_{a^{+}}^{p} f(b, x(b))-\sum_{i=1}^{m} \mu_{i}{ }^{\rho} \Im_{a^{+}}^{p+\xi_{i}} f\left(\delta_{i}, x\left(\delta_{i}\right)\right)-\varrho\right)
$$

Therefore, the results follow by substituting Eq. (3.14) in Eq. (3.8). The converse follows by simple calculation as above.

Denote by $\mathrm{C}=C([a, b], \mathbb{R})$ the Banach space of all continuous functions from $[a, b]$ to $\mathbb{R}$ defined by

$$
\|x\|=\sup _{a \leq t \leq b}|x(t)|,
$$

and define the operator $\mathbb{F}: \mathrm{C} \rightarrow \mathrm{C}$ by

$$
\begin{aligned}
(\mathbb{F} x)(t)= & \frac{\rho^{1-r}}{\Upsilon \Gamma(r)}\left(t^{\rho}-a^{\rho}\right)^{r-1}\left(\rho \mathfrak{I}_{a^{+}}^{p} f(s, x(s))(b)-\sum_{i=1}^{m} \mu_{i}^{\rho} \mathfrak{I}_{a^{+}}^{p+\xi_{i}} f(s, x(s))\left(\delta_{i}\right)-\varrho\right) \\
& +{ }^{\rho} \mathfrak{I}_{a^{+}}^{p} f(s, x(s))(t) .
\end{aligned}
$$

We show that the operator $\mathbb{F}$ has a fixed point which is the solutions of the problem (1.6). For simplicity, we denote

$$
\begin{aligned}
\Phi= & \frac{\rho^{1-(p+r)}\left(b^{\rho}-a^{\rho}\right)^{p+r-1}}{|\Upsilon| \Gamma(r) \Gamma(p+1)}+\frac{\rho^{1-r}\left(b^{\rho}-a^{\rho}\right)^{r-1}}{|\Upsilon| \Gamma(r)} \sum_{i=1}^{m}\left|\mu_{i}\right| \frac{\left(\delta_{i}^{\rho}-a^{\rho}\right)^{p+\xi_{i}}}{\rho^{p+\xi_{i}} \Gamma\left(p+\xi_{i}+1\right)} \\
& +\frac{\left(b^{\rho}-a^{\rho}\right)^{p}}{\rho^{p} \Gamma(p+1)}
\end{aligned}
$$




\subsection{Existence result via Banach contraction principle}

We prove first the existence and uniqueness result of the proposed problem (1.6) by applying the concept of the Banach contraction principle in this subsection. Thus, before we state the main theorem, we make the following assumptions:

$\left(T_{1}\right)$ Suppose that $f:[a, b] \times \mathbb{R} \rightarrow \mathbb{R}$ is a continuous functions such that $f(\cdot, x(\cdot)) \in \mathrm{C}$ for any $x \in \mathrm{C}$.

$\left(T_{2}\right)$ There exists a constant $\mathcal{A}>0$ such that

$$
|f(t, x)-f(t, y)| \leq \mathcal{A}|x-y|, \quad \text { for all } x, y \in \mathbb{R}, t \in[a, b] .
$$

Theorem 3.2 Suppose that assumptions $\left(T_{1}\right)-\left(T_{2}\right)$ are satisfied. Then problem (1.6) has a unique solution on $[a, b]$ provided that

$$
\mathcal{A} \Phi<1
$$

where $\Phi$ is defined by (3.16).

Proof Firstly, we construct a ball $\mathbb{B}_{\chi}=\{x \in \mathrm{C}:\|x\| \leq \chi\}$, where

$$
\chi \geq \frac{P \Phi+\frac{\rho^{1-r}\left(b^{\rho}-a^{\rho}\right)^{r-1}|\rho|}{|r| \Gamma(r)}}{1-\mathcal{A} \Phi}, \quad P=\sup _{t \in[a, b]}|f(t, 0)| .
$$

Then we show that $\mathbb{F} \mathbb{B}_{\chi} \subset \mathbb{B}_{\chi}$. Now, for any $x \in \mathbb{B}_{\chi}$, we have

$$
\begin{aligned}
|(\mathbb{F} x)(t)| \leq & \sup _{t \in[a, b]}\left\{\frac{\rho^{1-r}}{|\Upsilon| \Gamma(r)}\left(b^{\rho}-a^{\rho}\right)^{r-1} \rho \mathfrak{I}_{a^{+}}^{p}|f(s, x(s))|(b)\right. \\
& +\frac{\rho^{1-r}}{|\Upsilon| \Gamma(r)}\left(b^{\rho}-a^{\rho}\right)^{r-1} \sum_{i=1}^{m}\left|\mu_{i}\right|^{\rho} \mathfrak{J}_{a^{+}}^{p+\xi_{i}}|f(s, x(s))|\left(\delta_{i}\right) \\
& \left.+\frac{\rho^{1-r}}{|\Upsilon| \Gamma(r)}\left(b^{\rho}-a^{\rho}\right)^{r-1}|\varrho|+{ }^{\rho} \mathfrak{I}_{a^{+}}^{p}|f(s, x(s))|(t)\right\} \\
\leq & \frac{\rho^{1-r}}{|\Upsilon| \Gamma(r)}\left(b^{\rho}-a^{\rho}\right)^{r-1} \rho \mathfrak{J}_{a^{+}}^{p}(|f(s, x(s))-f(s, 0)|+|f(s, 0)|)(b) \\
& +\frac{\rho^{1-r}}{|\Upsilon| \Gamma(r)}\left(b^{\rho}-a^{\rho}\right)^{r-1} \sum_{i=1}^{m}\left|\mu_{i}\right|^{\rho} \mathfrak{I}_{a^{+}}^{p+\xi_{i}}(|f(s, x(s))-f(s, 0)|+|f(s, 0)|)\left(\delta_{i}\right) \\
& +\frac{\rho^{1-r}}{|\Upsilon| \Gamma(r)}\left(b^{\rho}-a^{\rho}\right)^{r-1}|\varrho|+{ }^{\rho} \mathfrak{I}_{a^{+}}^{p}(|f(s, x(s))-f(s, 0)|+|f(s, 0)|)(t) \\
\leq & (\mathcal{A}\|x\|+P)\left(\frac{\rho^{1-(p+r)}}{|\Upsilon| \Gamma(r) \Gamma(p+1)}\left(b^{\rho}-a^{\rho}\right)^{p+r-1}\right. \\
& \left.+\frac{\rho^{1-r}}{|\Upsilon| \Gamma(r)}\left(b^{\rho}-a^{\rho}\right)^{r-1} \sum_{i=1}^{m} \frac{\left|\mu_{i}\right|}{\Gamma\left(p+\xi_{i}+1\right)}\left(\frac{\delta_{i}^{\rho}-a^{\rho}}{\rho}\right)^{p+\xi_{i}}+\frac{\left(b^{\rho}-a^{\rho}\right)^{p}}{\rho^{p} \Gamma(p+1)}\right) \\
& +\frac{\rho^{1-r}}{|\Upsilon| \Gamma(r)}\left(b^{\rho}-a^{\rho}\right)^{r-1}|\varrho|
\end{aligned}
$$




$$
\begin{aligned}
& \leq(\mathcal{A}\|x\|+P) \Phi+\frac{\rho^{1-r}}{|\Upsilon| \Gamma(r)}\left(b^{\rho}-a^{\rho}\right)^{r-1}|\varrho| \\
& \leq \chi
\end{aligned}
$$

This shows that $\|(\mathbb{F})(x)\| \leq \chi$ and hence $\mathbb{F B}_{\chi} \subset \mathbb{B}_{\chi}$. Finally, we show that the operator $\mathbb{F}$ defined by (3.15) is contractive. Indeed, for any $x, y \in \mathbb{B}_{\chi}$, we have

$$
\begin{aligned}
|(\mathbb{F} x)(t)-(\mathbb{F} y)(t)| & \\
\leq & \frac{\rho^{1-r}}{|\Upsilon| \Gamma(r)}\left(b^{\rho}-a^{\rho}\right)^{r-1} \rho \mathfrak{J}_{a^{+}}^{p}(|f(s, x(s))-f(s, y(s))|)(b) \\
& +\frac{\rho^{1-r}}{|\Upsilon| \Gamma(r)}\left(b^{\rho}-a^{\rho}\right)^{r-1} \sum_{i=1}^{m}\left|\mu_{i}\right|^{\rho} \mathfrak{J}_{a^{+}}^{p+\xi_{i}}(|f(s, x(s))-f(s, y(s))|)\left(\delta_{i}\right) \\
& +{ }^{\rho} \mathfrak{I}_{a^{+}}^{p}(|f(s, x(s))-f(s, y(s))|)(t) \\
\leq & \mathcal{A}|x-y|\left(\frac{\rho^{1-(p+r)}}{|\Upsilon| \Gamma(r) \Gamma(p+1)}\left(b^{\rho}-a^{\rho}\right)^{p+r-1}\right. \\
\quad & \left.\frac{\rho^{1-r}}{|\Upsilon| \Gamma(r)}\left(b^{\rho}-a^{\rho}\right)^{r-1} \sum_{i=1}^{m} \frac{\left|\mu_{i}\right|}{\Gamma\left(p+\xi_{i}+1\right)}\left(\frac{\delta_{i}^{\rho}-a^{\rho}}{\rho}\right)^{p+\xi_{i}}+\frac{\left(b^{\rho}-a^{\rho}\right)^{p}}{\rho^{p} \Gamma(p+1)}\right) \\
\leq & \mathcal{A} \Phi|x-y| .
\end{aligned}
$$

Therefore, $\|\mathbb{F} x-\mathbb{F} y\| \leq \mathcal{A} \Phi\|x-y\|$. Thus in view of (3.17), the operator $\mathbb{F}$ is contractive and hence by the Banach contraction principle, problem (1.6) has a unique solution on $[a, b]$.

Remark 3.3 We now present some special cases of our Theorem 3.2. For instance, to obtain the result of [12] (see Theorem 3.2), we set the parameters $\rho=1$ and $\varrho=0$, then we obtain

$$
\Phi^{*}=\frac{(b-a)^{p+r-1}}{\left|\Upsilon^{*}\right| \Gamma(r) \Gamma(p+1)}+\frac{(b-a)^{r-1}}{\left|\Upsilon^{*}\right| \Gamma(r)} \sum_{i=1}^{m}\left|\mu_{i}\right| \frac{\left(\delta_{i}-a\right)^{p+\xi_{i}}}{\Gamma\left(p+\xi_{i}+1\right)}+\frac{(b-a)^{p}}{\Gamma(p+1)},
$$

where

$$
\Upsilon^{*}=\sum_{i=1}^{m} \frac{\mu_{i}}{\Gamma\left(r+\xi_{i}\right)}\left(\delta_{i}-a\right)^{r+\xi_{i}-1}-\frac{1}{\Gamma(r)}(b-a)^{r-1} .
$$

We now state the following corollary.

Corollary 3.4 Suppose that assumptions $\left(T_{1}\right)-\left(T_{2}\right)$ hold. Then problem (1.6) has a unique solution provided that $\mathcal{A} \Phi^{*}<1$.

Our next existence results are established using the concepts of Krasnoselskii's fixed point theorem. 


\subsection{Existence result via Krasnoselskii's fixed point theorem}

In this subsection, we establish the existence results via Kransnoselkii's fixed point theorem [30].

Theorem 3.5 ([30]) Let $P$ be a closed, convex, and nonempty subset of a Banach space $X$, and let $F_{1}, F_{2}$ be operators such that:

(i) $F_{1} y+F_{2} z \in M$ whenever $y, z \in P$.

(ii) $F_{1}$ is compact and continuous.

(iii) $F_{2}$ is a contraction mapping.

Then there exists $w \in P$ such that $w=F_{1} w+F_{2} w$.

Theorem 3.6 Suppose that $f:[a, b] \times \mathbb{R} \rightarrow \mathbb{R}$ is a continuous function fulfilling assumption $\left(T_{2}\right)$ and the following hypotheses are true:

$\left(T_{3}\right)|f(t, y)| \leq v(t)$, for all $(t, y) \in[a, b] \times \mathbb{R}$ and $v:[a, b] \rightarrow \mathbb{R}$ is a continuous function. Then problem (1.6) has at least one solution on $[a, b]$, on condition that

$$
\mathcal{A} \psi<1
$$

where

$$
\psi=\frac{\rho^{1-(p+r)}\left(b^{\rho}-a^{\rho}\right)^{p+r-1}}{|\Upsilon| \Gamma(r) \Gamma(p+1)}+\frac{\rho^{1-r}\left(b^{\rho}-a^{\rho}\right)^{r-1}}{|\Upsilon| \Gamma(r)} \sum_{i=1}^{m} \frac{\left|\mu_{i}\right|}{\Gamma\left(p+\xi_{i}+1\right)}\left(\frac{\delta_{i}^{\rho}-a^{\rho}}{\rho}\right)^{p+\xi_{i}}
$$

Proof Denoting $\|v\|=\sup _{t \in[a, b]}|v(t)|$ and selecting $d \geq\|v\| \Phi+\frac{\rho^{1-r}\left(b^{\rho}-a^{\rho}\right)^{r-1}}{|\gamma| \Gamma(r)}|\varrho|$ we consider a set $\mathcal{B}_{d}=\{x \in \mathcal{C}:\|x\| \leq d\}$. Splitting the operator $\mathbb{F}: \mathrm{C} \rightarrow \mathrm{C}$ defined by (3.15) on $\mathcal{B}_{d}$ as $\mathbb{F}=\mathcal{F}+\mathcal{G}$, where

$$
\mathcal{F} x(t)={ }^{\rho} \mathfrak{I}_{a^{+}}^{p+\xi_{i}} f(s, x(s))(t), \quad t \in[a, b],
$$

and

$$
\begin{aligned}
& \mathcal{G} x(t)=\frac{\rho^{1-r}}{\Upsilon \Gamma(r)}\left(t^{\rho}-a^{\rho}\right)^{r-1}\left(\rho \Im^{p} a^{+} f(s, x(s))(b)-\sum_{i=1}^{m} \mu_{i}^{\rho} \Im_{a^{+}}^{p+\xi_{i}} f(s, x(s))\left(\delta_{i}\right)-\varrho\right), \\
& t \in[a, b] .
\end{aligned}
$$

Step 1. We show that $\mathcal{F} x+\mathcal{G} y \in \mathcal{B}_{d}$. Indeed, for any $x, y \in \mathcal{B}_{d}$ and $t \in[a, b]$, we get

$$
\begin{aligned}
& |(\mathcal{F} x)(t)+(\mathcal{G} y)(t)| \\
& \leq \sup _{t \in[a, b]}\left\{{ }^{\rho} \Im_{a^{+}}^{p+\xi_{i}}|f(s, x(s))|(t)+\frac{\rho^{1-r}\left(t^{\rho}-a^{\rho}\right)^{r-1} \rho}{\Upsilon \Gamma(r)} \Im^{p} a^{+}|f(s, x(s))|(b)\right. \\
& \left.\quad+\frac{\rho^{1-r}\left(t^{\rho}-a^{\rho}\right)^{r-1}}{\Upsilon \Gamma(r)} \sum_{i=1}^{m}\left|\mu_{i}\right|^{\rho} \Im_{a^{+}}^{p+\xi_{i}}|f(s, x(s))|\left(\delta_{i}\right)+\frac{\rho^{1-r}\left(t^{\rho}-a^{\rho}\right)^{r-1}}{\Upsilon \Gamma(r)}|\varrho|\right\} \\
& \leq\|v\|\left(\frac{\rho^{1-(p+r)}}{|\Upsilon| \Gamma(r) \Gamma(p+1)}\left(b^{\rho}-a^{\rho}\right)^{p+r-1}\right.
\end{aligned}
$$




$$
\begin{aligned}
& \left.\quad+\frac{\rho^{1-r}}{|\Upsilon| \Gamma(r)}\left(b^{\rho}-a^{\rho}\right)^{r-1} \sum_{i=1}^{m} \frac{\left|\mu_{i}\right|}{\Gamma\left(p+\xi_{i}+1\right)}\left(\frac{\delta_{i}^{\rho}-a^{\rho}}{\rho}\right)^{p+\xi_{i}}+\frac{\left(b^{\rho}-a^{\rho}\right)^{p}}{\rho^{p} \Gamma(p+1)}\right) \\
& \quad+\frac{\rho^{1-r}}{|\Upsilon| \Gamma(r)}\left(b^{\rho}-a^{\rho}\right)^{r-1}|\varrho| \\
& \leq\|v\| \Phi+\frac{\rho^{1-r}}{|\Upsilon| \Gamma(r)}\left(b^{\rho}-a^{\rho}\right)^{r-1}|\varrho| \\
& \leq d .
\end{aligned}
$$

Therefore, $\|\mathcal{F} x+\mathcal{G} y\| \leq d$, which implies that $\mathcal{F} x+\mathcal{G} y \in \mathcal{B}_{d}$.

Step 2. We show that the operator $\mathcal{G}$ is a contraction.

Let $x, y \in \mathcal{B}_{d}$ and $t \in[a, b]$. We have

$$
\begin{aligned}
\|\mathcal{G} x-\mathcal{G} y\| \leq & \sup _{t \in[a, b]}\left\{\frac{\rho^{1-r}\left(t^{\rho}-a^{\rho}\right)^{r-1} \rho}{|\Upsilon| \Gamma(r)} \mathfrak{I}_{a^{+}}^{p}|f(s, x(s))-f(s, y(s))|(b)\right. \\
& \left.+\frac{\rho^{1-r}\left(t^{\rho}-a^{\rho}\right)^{r-1}}{|\Upsilon| \Gamma(r)} \sum_{i=1}^{m}\left|\mu_{i}\right|^{\rho} \mathfrak{J}_{a^{+}}^{p+\xi_{i}}|f(s, x(s))-f(s, y(s))|\left(\delta_{i}\right)\right\} \\
\leq & \mathcal{A}\left(\frac{\rho^{1-(p+r)}\left(b^{\rho}-a^{\rho}\right)^{p+r-1}}{|\Upsilon| \Gamma(r) \Gamma(p+1)}\right. \\
& \left.+\frac{\rho^{1-r}\left(b^{\rho}-a^{\rho}\right)^{r-1}}{|\Upsilon| \Gamma(r)} \sum_{i=1}^{m} \frac{\left|\mu_{i}\right|}{\Gamma\left(p+\xi_{i}+1\right)}\left(\frac{\delta_{i}^{\rho}-a^{\rho}}{\rho}\right)^{p+\xi_{i}}\right)\|x-y\| \\
\leq & \mathcal{A} \psi\|x-y\| .
\end{aligned}
$$

Thus, in view of condition (3.23), we conclude that the operator $\mathcal{G}$ is a contraction.

Step 3. We show that the operator $\mathcal{F}$ is compact and continuous.

Since the function $f \in C([a, b], \mathbb{R})$, the operator $\mathcal{F}$ is also continuous. Moreover, it is not difficult to see that the operator $\mathcal{F}$ is uniformly bounded. For any $x \in \mathcal{B}_{d}$, we have

$$
\|\mathcal{F} x\| \leq \frac{\left(b^{\rho}-a^{\rho}\right)^{p}}{\rho^{p} \Gamma(p+1)}\|v\|<\infty .
$$

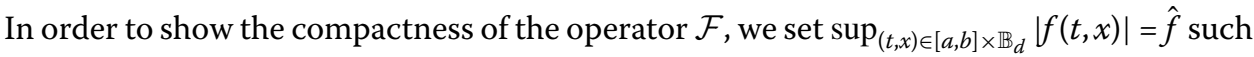
that, for any $t_{1}, t_{2} \in[a, b]$ with $t_{1}<t_{2}$, one has

$$
\begin{aligned}
&\left|(\mathcal{F} x)\left(t_{2}\right)-(\mathcal{F} x)\left(t_{1}\right)\right| \leq \mid \frac{\rho^{p}}{\Gamma(p)}\left(\int_{a}^{t_{1}} s^{\rho-1}\left[\left(t_{2}^{\rho}-s^{\rho}\right)^{p-1}-\left(t_{1}^{\rho}-s^{\rho}\right)^{p-1}\right] f(s, x(s)) d s\right. \\
&\left.+\int_{t_{1}}^{t_{2}} s^{\rho-1}\left(t_{2}^{\rho}-s^{\rho}\right)^{p-1} f(s, x(s)) d s\right) \mid \\
& \leq \frac{\hat{f}}{\rho^{p} \Gamma(p+a)}\left[2\left(t_{2}^{\rho}-t_{1}^{\rho}\right)^{p}+\left|\left(t_{2}-a\right)^{\rho p}-\left(t_{1}-a\right)^{\rho p}\right|\right] \\
& \rightarrow \infty, \quad \text { as } t_{2} \rightarrow t_{1} .
\end{aligned}
$$

This shows that the operator $\mathcal{F}$ is equicontinuous and, therefore, is relatively compact on $\mathcal{B}_{d}$. Thus, as a result of the Arzelá-Ascoli theorem, it follows that $\mathcal{F}$ is compact on 
$\mathcal{B}_{d}$. Since all the hypotheses of Theorem 3.5 are fulfilled, problem (1.6) has at least one solution on $[a, b]$.

Remark 3.7 We now present a special case of Theorem 3.2. For instance, to obtained the result of [12] (see Theorem 3.17), we set the parameters $\rho=1$ and $\varrho=0$, then we obtain

$$
\psi^{*}=\frac{(b-a)^{p+r-1}}{\left|\Upsilon^{*}\right| \Gamma(r) \Gamma(p+1)}+\frac{(b-a)^{r-1}}{\left|\Upsilon^{*}\right| \Gamma(r)} \sum_{i=1}^{m}\left|\mu_{i}\right| \frac{\left(\delta_{i}-a\right)^{p+\xi_{i}}}{\Gamma\left(p+\xi_{i}+1\right)}
$$

where

$$
\Upsilon^{*}=\sum_{i=1}^{m} \frac{\mu_{i}}{\Gamma\left(r+\xi_{i}\right)}\left(\delta_{i}-a\right)^{r+\xi_{i}-1}-\frac{1}{\Gamma(r)}(b-a)^{r-1} .
$$

We now state the following corollary.

Corollary 3.8 Suppose that assumptions $\left(T_{1}\right)-\left(T_{3}\right)$ hold. Then problem (1.6) has at least one solution on $[a, b]$, provided that $\mathcal{A} \psi^{*}<1$.

\section{Stability results}

In this section, we present two different types of results for stability, namely Ulam-Hyers and generalized Ulam-Hyers stability, respectively. Before we state the main theorem, the following definitions are needed.

Let $\epsilon>0$ and $\theta:[a, b] \rightarrow[a, \infty]$ be a continuous function. We consider the following inequalities:

$$
\begin{aligned}
& \left|{ }^{\rho} \mathfrak{D}_{a^{+}}^{p, q} y(t)-f(t, y(t))\right| \leq \epsilon, \quad t \in[a, b], \\
& \left|{ }^{\rho} \mathfrak{D}_{a^{+}}^{p, q} y(t)-f(t, y(t))\right| \leq \epsilon \theta(t), \quad t \in[a, b] .
\end{aligned}
$$

Definition 4.1 Problem (1.6) is Ulam-Hyers stable if there exists a constant $\tau>0$ such that, for $\epsilon>0$ and for each solution $y \in \mathrm{C}$ of the inequality (4.1), there exists a solution $x \in \mathrm{C}$ of problem (1.6) with

$$
|y(t)-x(t)| \leq \tau \epsilon, \quad t \in[a, b] .
$$

Definition 4.2 Problem (1.6) is generalized Ulam-Hyers stable if there exists $\theta_{f} \in$ $C\left(\mathbb{R}^{+}, \mathbb{R}^{+}\right)$and $\theta_{f}(0)=0$ such that, for each solution $y \in C$ of the inequality (4.2), there exists a solution $x \in \mathrm{C}$ of problem (1.6) with

$$
|y(t)-x(t)| \leq \theta_{f}(\epsilon), \quad t \in[a, b] .
$$

Remark 4.3 A function $y \in \mathrm{C}$ is a solution of the equality (4.1) if and only if there exists a function $z \in \mathrm{C}$ (which depends on $\mathrm{y}$ ) such that:

(i) $|z(t)| \leq \epsilon, t \in[a, b]$.

(ii) ${ }^{\rho} \mathfrak{D}_{a^{+}}^{p, q} y(t)=f(t, y(t))+z(t), t \in[a, b]$. 
Lemma 4.4 Let $1<r \leq 2,0 \leq p \leq 1$, if a function $y \in \mathrm{C}$ is a solution of the inequality (4.1), then $y$ is a solution of the following integral inequality:

$$
\left|y(t)-E_{y}-{ }^{\rho} \widetilde{I}_{a^{+}}^{p} f(s, y(s))(t)\right| \leq \Phi \epsilon,
$$

where

$$
E_{y}=\frac{\rho^{1-r}\left(t^{\rho}-a^{\rho}\right)^{r-1}}{\Upsilon \Gamma(r)}{ }^{\rho} \Im_{a^{+}}^{p} f(s, y(s))(b)+\frac{\rho^{1-r}\left(t^{\rho}-a^{\rho}\right)^{r-1}}{\Upsilon \Gamma(r)} \sum_{i=1}^{m} \mu_{i}^{\rho} \mathfrak{I}_{a^{+}}^{p+\xi_{i}} f(s, y(s))\left(\delta_{i}\right) .
$$

Proof Using Lemma 3.1 and Remark 4.3, it follows that

$$
\begin{aligned}
y(t)= & \frac{\rho^{1-r}\left(t^{\rho}-a^{\rho}\right)^{r-1}}{\Upsilon \Gamma(r)}{ }^{\rho} \Im_{a^{+}}^{p} f(s, y(s))(b)-\frac{\rho^{1-r}\left(t^{\rho}-a^{\rho}\right)^{r-1}}{\Upsilon \Gamma(r)} \sum_{i=1}^{m} \mu_{i}^{\rho} \Im^{p+\xi_{a^{+}}} f(s, y(s))\left(\delta_{i}\right) \\
& -\frac{\rho^{1-r}\left(t^{\rho}-a^{\rho}\right)^{r-1}}{\Upsilon \Gamma(r)} \varrho+{ }^{\rho} \Im_{a^{+}}^{p} f(s, y(s))(t)+\frac{\rho^{1-r}\left(t^{\rho}-a^{\rho}\right)^{r-1}}{\Upsilon \Gamma(r)}{ }^{\rho} \Im_{a^{+}}^{p} z(b) \\
& -\frac{\rho^{1-r}\left(t^{\rho}-a^{\rho}\right)^{r-1}}{\Upsilon \Gamma(r)} \sum_{i=1}^{m} \mu_{i}^{\rho} \Im^{p+\xi_{i}} z\left(\delta_{i}\right)-\frac{\rho^{1-r}\left(t^{\rho}-a^{\rho}\right)^{r-1}}{\Upsilon \Gamma(r)} \varrho+{ }^{\rho} \Im_{a^{+}}^{p} z(t),
\end{aligned}
$$

which implies that

$$
\begin{aligned}
\mid y(t) & -E_{y}-{ }^{\rho} \mathfrak{I}_{a^{+}}^{p} f(s, y(s))(t) \mid \\
= & \mid \frac{\rho^{1-r}\left(t^{\rho}-a^{\rho}\right)^{r-1}}{\Upsilon \Gamma(r)}{ }^{\rho} \mathfrak{I}_{a^{+}}^{p} z(b) \\
& -\frac{\rho^{1-r}\left(t^{\rho}-a^{\rho}\right)^{r-1}}{\Upsilon \Gamma(r)} \sum_{i=1}^{m} \mu_{i}^{\rho} \mathfrak{I}_{a^{+}}^{p+\xi_{i}} z\left(\delta_{i}\right)-\frac{\rho^{1-r}\left(t^{\rho}-a^{\rho}\right)^{r-1}}{\Upsilon \Gamma(r)} \varrho+{ }^{\rho} \mathfrak{J}_{a^{+}}^{p} z(t) \mid \\
\leq & \epsilon\left(\frac{\rho^{1-(p+r)}}{|\Upsilon| \Gamma(r) \Gamma(p+1)}\left(b^{\rho}-a^{\rho}\right)^{p+r-1}\right. \\
& \left.+\frac{\rho^{1-r}}{|\Upsilon| \Gamma(r)}\left(b^{\rho}-a^{\rho}\right)^{r-1} \sum_{i=1}^{m} \frac{\left|\mu_{i}\right|}{\Gamma\left(p+\xi_{i}+1\right)}\left(\frac{\delta_{i}^{\rho}-a^{\rho}}{\rho}\right)^{p+\xi_{i}}+\frac{\left(b^{\rho}-a^{\rho}\right)^{p}}{\rho^{p} \Gamma(p+1)}\right) \\
\leq & \Phi \epsilon .
\end{aligned}
$$

We now state the main theorem as follows:

Theorem 4.5 Assume that hypotheses $\left(T_{1}\right)$ and $\left(T_{2}\right)$ are satisfied with $\mathcal{A} \Phi<1$. Then problem (1.6) is Ulam-Hyers stable and accordingly is generalized Ulam-Hyers stable.

Proof Suppose that $y \in \mathcal{C}$ is a solution of inequality (4.1) and $x \in \mathcal{C}$ is a unique solution of problem (1.6). Then it follows from Lemma 4.1 that

$$
\begin{aligned}
|y(t)-x(t)|= & \mid y(t)-\frac{\rho^{1-r}\left(t^{\rho}-a^{\rho}\right)^{r-1}}{\Upsilon \Gamma(r)}{ }^{\rho} \Im_{a^{+}}^{p} f(s, x(s))(b) \\
& -\frac{\rho^{1-r}\left(t^{\rho}-a^{\rho}\right)^{r-1}}{\Upsilon \Gamma(r)} \sum_{i=1}^{m} \mu_{i}^{\rho} \mathfrak{I}_{a^{+}}^{p+\xi_{i}} f(s, x(s))\left(\delta_{i}\right)-\frac{\rho^{1-r}\left(t^{\rho}-a^{\rho}\right)^{r-1}}{\Upsilon \Gamma(r)} \varrho
\end{aligned}
$$




$$
\begin{aligned}
& +{ }^{\rho} \mathfrak{I}_{a^{+}}^{p} f(s, x(s))(t) \mid \\
\leq & \left|y(t)-E_{y}-{ }^{\rho} \mathfrak{I}_{a^{+}}^{p} f(s, y(s))(t)\right| \\
& +\mid \frac{\rho^{1-r}\left(t^{\rho}-a^{\rho}\right)^{r-1}}{\Upsilon \Gamma(r)}{ }^{\rho} \Im_{a^{+}}^{p}(f(s, y(s))-f(s, x(s)))(b) \\
& -\frac{\rho^{1-r}\left(t^{\rho}-a^{\rho}\right)^{r-1}}{\Upsilon \Gamma(r)} \sum_{i=1}^{m} \mu_{i}^{\rho} \mathfrak{I}_{a^{+}}^{p+\xi_{i}}(f(s, y(s))-f(s, x(s)))\left(\delta_{i}\right) \\
& +{ }^{\rho} \mathfrak{I}_{a^{+}}^{p}(f(s, y(s))-f(s, x(s))(t)) \mid \\
\leq & \Phi \epsilon+\mathcal{A} \Phi|y(t)-x(t)|,
\end{aligned}
$$

which implies that

$$
\|y-x\| \leq \tau \epsilon
$$

where

$$
\tau=\frac{\Phi}{1-\mathcal{A} \Phi}>0
$$

Hence, we conclude that problem (1.6) is Ulam-Hyers stable. In addition, denoting $\theta_{f}(\epsilon)=$ $\tau \epsilon$, such that $\theta_{f}(0)=0$, then problem (1.6) is generalized Ulam-Hyers stable.

\section{Examples}

Example 5.1 Consider the Hilfer fractional differential equation with nonlocal boundary condition of the form

$$
\left\{\begin{array}{l}
1 \mathfrak{D}_{\frac{1}{3}}^{\frac{4}{3}, \frac{1}{2}} x(t)=\frac{1}{3\left(2^{3 t}+1\right)}\left(\frac{|x(t)|}{1+|x(t)|}\right)+\frac{3}{2}, \quad t \in\left[\frac{1}{3}, 3\right], \\
x(1)=0, \quad x(3)=\sqrt{2} \mathfrak{I}_{\frac{1}{3}}^{\frac{3}{2}} x\left(\frac{9}{4}\right)+\frac{5}{3} 1 \mathfrak{I}_{\frac{1}{3}}^{\frac{3}{5}} x\left(\frac{8}{3}\right)+5^{1} \mathfrak{I}_{\frac{1}{3}}^{\frac{2}{3}} x\left(\frac{7}{4}\right) .
\end{array}\right.
$$

By comparing problems (1.6) with (5.1), it is not difficult to figure out the following values: $\rho=1, p=\frac{4}{3}, q=\frac{1}{2}, r=\frac{5}{3}, a=\frac{1}{3}, b=3, \mu_{1}=\sqrt{2}, \mu_{2}=\frac{5}{3}, \mu_{3}=5, \xi_{1}=\frac{3}{2}, \xi_{2}=\frac{3}{5}, \xi_{3}=\frac{2}{3}$, $\delta_{1}=\frac{9}{4}, \delta_{2}=\frac{8}{3}, \delta_{3}=\frac{7}{4}, \varrho=0, m=1,2,3$. Obviously, for any $w \in \mathbb{R}$ and $t \in\left[\frac{1}{3}, 3\right]$, the function $f:\left[\frac{1}{3}, 3\right] \times \mathbb{R} \rightarrow \mathbb{R}$ with

$$
f(t, w)=\frac{1}{3\left(2^{3 t}+1\right)}\left(\frac{|w|}{1+|w|}\right)+\frac{3}{2}
$$

is a continuous function and, for all $w, \bar{w} \in \mathbb{R}_{+}$and $t \in\left[\frac{1}{3}, 3\right]$, this yields

$$
|f(t, w)-f(t, \bar{w})| \leq \frac{1}{9}(|w-\bar{w}|) .
$$

Consequently, it follows that assumptions $\left(T_{1}\right)$ and $\left(T_{2}\right)$ are satisfied with $\mathcal{A}=\frac{1}{9}$. Moreover, by simple calculations, we get $|\Upsilon| \approx 11.2817, \Phi=5.8525>0, \psi=2.7466>0$ and

$$
\mathcal{A} \Phi \approx 0.6503<1
$$


which implies that all the assumptions of Theorem 3.2 are satisfied. Hence, problem (1.6) has a unique solution on $\left[\frac{1}{3}, 3\right]$. Furthermore,

$$
\mathcal{A} \psi \approx 0.3052<1
$$

this shows that all hypotheses of Theorem 3.6 also hold, thus problem (1.6) has at least one solution on $\left[\frac{1}{3}, 3\right]$. In addition, we can see that $\tau \approx 16.7358>0$. So, according to Theorem 4.5, problem (1.6) is both Ulam-Hyers and generalized Ulam-Hyers stable.

Example 5.2 Consider the generalized nonlocal boundary value problem which involves the Hilfer-Katugampola fractional derivative described by

$$
\left\{\begin{array}{l}
\frac{1}{2} \mathfrak{D}_{0^{+}}^{\frac{5}{2}, \frac{2}{3}} x(t)=f(t, x(t)), \quad t \in[0,1], \\
x(0)=0, \quad x(1)=\frac{2}{3} \frac{1}{2} \mathfrak{I}_{0^{+}}^{\frac{5}{4}} x\left(\frac{1}{3}\right)+\frac{7}{3} \frac{1}{2} \mathfrak{I}_{0^{+}}^{\frac{4}{3}} x\left(\frac{2}{5}\right)+2 .
\end{array}\right.
$$

Here $\rho=\frac{1}{2}, p=\frac{5}{2}, q=\frac{2}{3}, r=\frac{3}{2}, a=0, b=1, \mu_{1}=\frac{2}{3}, \mu_{2}=\frac{7}{3}, \xi_{1}=\frac{5}{4}, \xi_{2}=\frac{4}{3}, \delta_{1}=\frac{1}{3}, \delta_{2}=\frac{2}{5}$, $\varrho=2, m=1,2$. By substituting these values in Eqs. (3.4), (3.16) and (3.23), we obtain

$$
|\Upsilon| \approx 1.0188, \quad \Phi \approx 12.2772>0 \text { and } \quad \psi \approx 10.5754>0
$$

Denote $f(t, x(t))=\frac{e^{2 t}+\cos (2 t)}{\left(4+3^{t}\right)}\left(\frac{|x(t)|}{1+|x(t)|}\right)+\frac{\sqrt{3}}{2}$. Then, for any $u, \bar{u} \in \mathbb{R}_{+}$and $t \in[0,1]$,

$$
|f(t, u)-f(t, \bar{u})| \leq \frac{1}{25}(|u-\bar{u}|)
$$

So assumptions $\left(T_{1}\right)$ and $\left(T_{2}\right)$ are fulfilled with $\mathcal{A}=\frac{1}{25}$ and $\mathcal{A} \Phi \approx 0.4911<1$. Therefore, all the assumptions of Theorem 3.2 are satisfied and problem (1.6) has a unique solution on $[0,1]$. Moreover, we can see that $\mathcal{A} \psi \approx 0.4230<1$. Thus, all the hypotheses of Theorem 3.6 hold. Hence, problem (1.6) has at least one solution on $[0,1]$.

\section{Conclusions}

In this article, we have shown that the proposed problem (1.6) is equivalent to the Volterra integral equation. Based on this equation, we have provided some sufficient conditions for the existence and uniqueness of solutions by utilizing the concepts of the Banach and Krasnoselskii's fixed point theorems. Also, we derive stability in the case of Ulam-Hyers and generalized Ulam-Hyers, respectively. Furthermore, two examples are given as an application to validate the results obtained. Also, it enables us to make the accompanying comments:

- If $\rho \rightarrow 1, \varrho=0$ problem (1.6) reduces to the problem considered in [12].

- Setting $0<p<1, \rho \rightarrow 1, \varrho=0$ and $\xi_{i}=0, i=1, \ldots, m$, problem (1.6) reduces to the problem considered in [48].

- Setting $0<p<1, \varrho=0$ and $\xi_{i}=0, i=1, \ldots, m$, problem (1.6) reduces to the problem considered in [20].

- If $\rho \rightarrow 0, \varrho=0$ and $\xi_{i}=0$ for all $i=1, \ldots, m$, the generalized nonlocal Riemann-Liouville integral condition reduces to the one considered in $[20,44,48,49]$. 
- If $\xi_{i}=1$ for all $i=1, \ldots, m$, the generalized nonlocal Riemann-Liouville integral condition takes the form

$$
x(b)=\mu_{1} \int_{a}^{\delta_{1}} x(\tau) d \tau+\mu_{2} \int_{a}^{\delta_{2}} x(\tau) d \tau+\cdots+\mu_{m} \int_{a}^{\delta_{m}} x(\tau) d \tau+\varrho,
$$

which plays a significant role in computational fluid dynamics, ill-posed problems and mathematical models and yields a better result than the initial condition $x(b)=x_{b}$ $[14,34]$.

Therefore, based on this setting, we conclude that our results are new and extend the above cited results which can be considered as a further development of the qualitative analysis of fractional differential equations. The analysis of the Ulam-Hyers-Mittag-Leffler function is to be discussed shortly in the context of Hilfer-Katugampola fractional derivative with generalized nonlocal conditions proposed in this paper or another mixed boundary condition.

\section{Acknowledgements}

The authors acknowledge the financial support provided by the Center of Excellence in Theoretical and Computational Science (TaCS-CoE), KMUTT. The first author was supported by the "Petchra Pra Jom Klao Ph.D. Research Scholarship from King Mongkut's University of Technology Thonburi" (Grant No. 13/2561). Also, the King Mongkut's University of

Technology Thonburi financially funded this research work through the KMUTT 55th Anniversary Commemorative Fund.

\section{Funding}

Petchra Pra Jom Klao Doctoral Scholarship for Ph.D. program of King Mongkut's University of Technology Thonburi (KMUTT). The Center of Excellence in Theoretical and Computational Science (TaCS-CoE), KMUTT. Center of Excellence in Theoretical and Computational Science (TaCS-CoE), KMUTT. King Mongkut's University of Technology North Bangkok, Contract no. KMUTNB-63-KNOW-033.

Availability of data and materials

Not applicable.

\section{Competing interests}

The authors declare to have no conflict of interest.

\section{Authors' contributions}

The authors contributed equally in writing this article. All authors read and approved the final manuscripts.

\section{Author details}

${ }^{1}$ KMUTTFixed Point Research Laboratory, Room SCL 802 Fixed Point Laboratory, Science Laboratory Building, Department of Mathematics, Faculty of Science, King Mongkut's University of Technology Thonburi (KMUTT), Bangkok, Thailand. ${ }^{2}$ Center of Excellence in Theoretical and Computational Science (TaCS-CoE), Science Laboratory Building, King Mongkut's University of Technology Thonburi (KMUTT), Bangkok, Thailand. ${ }^{3}$ Department of Mathematics and Computer Science, Sule Lamido University, Kafin-Hausa, Nigeria. ${ }^{4}$ Department of Medical Research, China Medical University Hospital, China Medical University, Taichung, Taiwan. ${ }^{5}$ Department of Mathematics, Faculty of Arts and Sciences, Cankaya University, Ankara, Turkey. ${ }^{6}$ Intelligent and Nonlinear Dynamic Innovations Research Center, Department of Mathematics, Faculty of Applied Science, King Mongkut's University of Technology North Bangkok (KMUTNB), Bangkok, Thailand. ${ }^{7}$ School of Continuing Education, Bayero University Kano, Kano, Nigeria.

\section{Publisher's Note}

Springer Nature remains neutral with regard to jurisdictional claims in published maps and institutional affiliations.

Received: 28 January 2020 Accepted: 4 May 2020 Published online: 19 May 2020

\section{References}

1. Abbas, S., Arifi, N.A., Benchohra, M., Zhou, Y.: Random coupled Hilfer and Hadamard fractional differential systems in generalized Banach spaces. Mathematics 7(3), 285 (2019)

2. Abbas, S., Benchohra, M., Lagreg, J., Alsaedi, A., Zhou, Y.: Existence and Ulam stability for fractional differential equations of Hilfer-Hadamard type. Adv. Differ. Equ. 2017(1), 180 (2017)

3. Abdeljawad, T., Jarad, F., Baleanu, D.: On the existence and the uniqueness theorem for fractional differential equations with bounded delay within Caputo derivatives. Sci. China Ser. A, Math. 51(10), 1775-1786 (2008)

4. Abdo, M.S., Panchal, S.K., Bhairat, S.P.: Existence of solution for Hilfer fractional differential equations with boundary value conditions. arXiv preprint (2019). arXiv:1909.13680 
5. Ahmad, B., Nieto, J..: Existence results for nonlinear boundary value problems of fractional integrodifferential equations with integral boundary conditions. Bound. Value Probl. 2009(1), 708576 (2009)

6. Ahmed, I., Kumam, P., Shah, K., Borisut, P., Sitthithakerngkiet, K., Demba, M.A.: Stability results for implicit fractional pantograph differential equations via $\phi$-Hilfer fractional derivative with a nonlocal Riemann-Liouville fractional integral condition. Mathematics 8(1), 94 (2020)

7. Ali, A., Shah, K., Jarad, F., et al.: Ulam-Hyers stability analysis to a class of nonlinear implicit impulsive fractional differential equations with three point boundary conditions. Adv. Differ. Equ. 2019(1), 7 (2019)

8. Ali, A., Shah, K., Jarad, F., Gupta, V., Abdeljawad, T.: Existence and stability analysis to a coupled system of implicit type impulsive boundary value problems of fractional-order differential equations. Adv. Differ. Equ. 2019(1), 101 (2019)

9. Alsaedi, A., Alghanmi, M., Ahmad, B., Ntouyas, S.: Generalized Liouville-Caputo fractional differential equations and inclusions with nonlocal generalized fractional integral and multipoint boundary conditions. Symmetry 10(12), 667 (2018)

10. Ameen, R., Jarad, F., Abdeljawad, T.: Ulam stability for delay fractional differential equations with a generalized Caputo derivative. Filomat 32(15), 5265-5274 (2018)

11. Aphithana, A., Ntouyas, S.K., Tariboon, J.: Existence and Ulam-Hyers stability for Caputo conformable differential equations with four-point integral conditions. Adv. Differ. Equ. 2019(1), 139 (2019)

12. Asawasamrit, S., Kijjathanakorn, A., Ntouyas, S.K., Tariboon, J.: Nonlocal boundary value problems for Hilfer fractional differential equations. Bull. Korean Math. Soc. 55(6), 1639-1657 (2018)

13. Borisut, P., Kumam, P., Ahmed, I., Sitthithakerngkiet, K.: Nonlinear Caputo fractional derivative with nonlocal Riemann-Liouville fractional integral condition via fixed point theorems. Symmetry 11(6), 829 (2019)

14. Ciegis, R., Bugajev, A.: Numerical approximation of one model of bacterial self-organization. Nonlinear Anal., Model. Control 17(3), 253-270 (2012)

15. Furati, K.M., Kassim, M.D., et al.: Existence and uniqueness for a problem involving Hilfer fractional derivative. Comput. Math. Appl. 64(6), 1616-1626 (2012)

16. Gambo, Y, Ameen, R., Jarad, F, Abdeljawad, $T$. Existence and uniqueness of solutions to fractional differential equations in the frame of generalized Caputo fractional derivatives. Adv. Differ. Equ. 2018(1), 134 (2018)

17. Gambo, Y.Y., Jarad, F., Baleanu, D., Abdeljawad, T.: On Caputo modification of the Hadamard fractional derivatives. Adv. Differ. Equ. 2014(1), 10 (2014)

18. Gerolymatou, E., Vardoulakis, I., Hilfer, R.: Modelling infiltration by means of a nonlinear fractional diffusion model. J. Phys. D, Appl. Phys. 39(18), 4104 (2006)

19. Harikrishnan, S., Ibrahim, R., Kanagarajan, K.: Establishing the existence of Hilfer fractional pantograph equations with impulses. Fundam. J. Math. Appl. 1(1), 36-42 (2018)

20. Harikrishnan, S., Kanagarajan, K., Elsayed, E.: Existence of solutions of nonlocal initial value problems for differential equations with Hilfer-Katugampola fractional derivative. Rev. R. Acad. Cienc. Exactas Fís. Nat., Ser. A Mat. (2019). https://doi.org/10.1007/s13398-019-00645-0

21. Harikrishnan, S., Shah, K., Baleanu, D., Kanagarajan, K.: Note on the solution of random differential equations via $\psi$-Hilfer fractional derivative. Adv. Differ. Equ. 2018(1), 224 (2018)

22. Hyers, D.H.: On the stability of the linear functional equation. Proc. Natl. Acad. Sci. USA 27(4), 222 (1941)

23. Jarad, F., Abdeljawad, T:: A modified Laplace transform for certain generalized fractional operators. Results Nonlinear Anal. 1(2), 88-98 (2018)

24. Jarad, F., Abdeljawad, T.: Variational principles in the frame of certain generalized fractional derivatives. Discrete Contin. Dyn. Syst., Ser. S 13(3), 695-708 (2020)

25. Jarad, F., Abdeljawad, T., Baleanu, D.: Caputo-type modification of the Hadamard fractional derivatives. Adv. Differ. Equ. 2012(1), 142 (2012)

26. Jarad, F., Abdeljawad, T., Baleanu, D.: On the generalized fractional derivatives and their Caputo modification. J. Nonlinear Sci. Appl. 10(5), 2607-2619 (2017)

27. Jarad, F., Abdeljawad, T., Hammouch, Z:: On a class of ordinary differential equations in the frame of Atangana-Baleanu fractional derivative. Chaos Solitons Fractals 117, 16-20 (2018)

28. Jarad, F., Harikrishnan, S., Shah, K., Kanagarajan, K.: Existence and stability results to a class of fractional random implicit differential equations involving a generalized Hilfer fractional derivative. Discrete Contin. Dyn. Syst., Ser. S 13(3), 723-739 (2020)

29. Kilbas, A., Srivastava, H., Trujillo, J.: Theory and Applications of Fractional Derivatial Equations. North-Holland Mathematics Studies, vol. 204 (2006)

30. Krasnoselskii, M.: Two remarks about the method of successive approximations. Mat. Nauk 10, 123-127 (1955)

31. Luo, D., Shah, K., Luo, Z:: On the novel Ulam-Hyers stability for a class of nonlinear $\psi$-Hilfer fractional differential equation with time-varying delays. Mediterr. J. Math. 16(5), 112 (2019)

32. Mainardi, F.: Fractional Calculus and Waves in Linear Viscoelasticity: An Introduction to Mathematical Models. World Scientific, Singapore (2010)

33. Nieto, J., Ouahab, A., Venktesh, V: Implicit fractional differential equations via the Liouville-Caputo derivative. Mathematics 3(2), 398-411 (2015)

34. Ntouyas, S.: Nonlocal initial and boundary value problems: a survey. In: Handbook of Differential Equations: Ordinary Differential Equations, vol. 2, pp. 461-557. Elsevier, Amsterdam (2006)

35. Oliveira, D., de Oliveira, E.C.: Hilfer-Katugampola fractional derivatives. Comput. Appl. Math. 37(3), 3672-3690 (2018)

36. Osler, T.J.: The fractional derivative of a composite function. SIAM J. Math. Anal. 1(2), 288-293 (1970)

37. Podlubny, I.: Fractional Differential Equations: An Introduction to Fractional Derivatives, Fractional Differentia Equations, to Methods of Their Solution and Some of Their Applications, vol. 198. Elsevier, Amsterdam (1998)

38. Rassias, T.M.: On the stability of the linear mapping in Banach spaces. Proc. Am. Math. Soc. 72(2), 297-300 (1978)

39. Samko, S.G., Kilbas, A.A., Marichev, O.I., et al.. Fractional Integrals and Derivatives, vol. 1993. Gordon \& Breach, Yverdon (1993)

40. Shah, K., Ali, A., Bushnaq, S.: Hyers-Ulam stability analysis to implicit Cauchy problem of fractional differential equations with impulsive conditions. Math. Methods Appl. Sci. 41(17), 8329-8343 (2018)

41. Shah, K., Hussain, W.: Investigating a class of nonlinear fractional differential equations and its Hyers-Ulam stability by means of topological degree theory. Numer. Funct. Anal. Optim. 40(12), 1355-1372 (2019) 
42. Srivastava, H., El-Sayed, A., Gaafar, F.: A class of nonlinear boundary value problems for an arbitrary fractional-order differential equation with the Riemann-Stieltjes functional integral and infinite-point boundary conditions. Symmetry 10(10), 508 (2018)

43. Srivastava, H.M., Das, A., Hazarika, B., Mohiuddine, S.: Existence of solution for non-linear functional integral equations of two variables in Banach algebra. Symmetry 11(5), 674 (2019)

44. Staněk, S.: Existence results for implicit fractional differential equations with nonlocal boundary conditions. Mem. Differ. Equ. Math. Phys. 72, 119-130 (2017)

45. Tomovski, Ž., Hilfer, R., Srivastava, H.M.: Fractional and operational calculus with generalized fractional derivative operators and Mittag-Leffler type functions. Integral Transforms Spec. Funct. 21(11), 797-814 (2010)

46. Ulam, S.M.: A Collection of Mathematical Problems, vol. 8. Interscience, New York (1960)

47. Ulam, S.M.: Problems in Modern Mathematics. Courier Corporation, Chelmsford (2004)

48. Vivek, D., Kanagarajan, K., Elsayed, E.: Some existence and stability results for Hilfer-fractional implicit differential equations with nonlocal conditions. Mediterr. J. Math. 15(1), 15 (2018)

49. Vivek, D., Kanagarajan, K., Harikrishnan, S.: Analytic study on nonlocal initial value problems for pantograph equations with Hilfer-Hadamard fractional derivative. rn 55, 7 (2018)

50. Vivek, D., Kanagarajan, K., Sivasundaram, S.: Dynamics and stability of pantograph equations via Hilfer fractional derivative. Nonlinear Stud. 23, 4 (2016)

51. Wang, C., Xu, T.-Z.: Stability of the nonlinear fractional differential equations with the right-sided Riemann-Liouville fractional derivative. Discrete Contin. Dyn. Syst., Ser. S 10, 3 (2017)

52. Wang, G., Ghanmi, A., Horrigue, S., Madian, S.: Existence result and uniqueness for some fractional problem. Mathematics 7(6), 516 (2019)

53. Wang, J., Zhang, Y.: Nonlocal initial value problems for differential equations with Hilfer fractional derivative. Appl. Math. Comput. 266, 850-859 (2015)

54. Yong, Z., Jinrong, W., Lu, Z.: Basic Theory of Fractional Differential Equations. World Scientific, Singapore (2016)

55. Zhang, W., Liu, W., Xue, T.: Existence and uniqueness results for the coupled systems of implicit fractional differential equations with periodic boundary conditions. Adv. Differ. Equ. 2018(1), 413 (2018)

\section{Submit your manuscript to a SpringerOpen ${ }^{\circ}$ journal and benefit from:}

- Convenient online submission

Rigorous peer review

- Open access: articles freely available online

- High visibility within the field

- Retaining the copyright to your article

Submit your next manuscript at $\boldsymbol{~ s p r i n g e r o p e n . c o m ~}$ 